\section{Posterior headache as a warning symptom of vertebral dissection: a case report}

\author{
S. Lanfranchi • M. Di Falco • M. Perini \\ D. Zarcone \\ U.O. Neurologia, \\ Azienda Ospedaliera "S. Antonio Abate", \\ Via Pastori 4, Gallarate (VA), Italy \\ e-mail: stefy.lanfranchi@libero.it \\ Tel.: +39-0331-751165 \\ Fax: 39-0331-751762
}

Published online: 15 December 2005

\begin{abstract}
Cervicocerebral arterial dissection is an important cause of stroke in young adults; the onset is often characterised by severe occipital headache, followed by nausea, vomiting and vertigo, mimicking a migraine attack. We describe herewith a case of vertebral arterial dissection with cerebellar infarction, which started with a posterior headache and neurovegetative symptoms, without other signs. Recommendations for recognition of similar cases, potentially dangerous and treatable, are discussed.
\end{abstract}

Keywords Cervicocerebral arterial dissections (CAD) • Headache $\cdot$ Stroke

\section{Introduction}

Cervicocerebral arterial dissections (CAD) are an important cause of strokes in young adults, accounting for nearly $20 \%$ of strokes in patients under 45 -years-old; vertebral artery (VA) dissections account for about $15 \%$ of all CAD [1]. The aetiopathogenesis is not completely understood, though neck trauma, whiplash, infections and underlying arteriopathy are considered important [2]. Severe posterior headache and neurovegetative symptoms with nausea and vomiting, mimicking a migraine attack, are the more frequent manifestations [3]. An early clinical diagnosis is mandatory to start proper treatment and achieve the best possible outcome.

\section{Case report}

A 55-year-old man with a previous history of bone neoplasm 12 years earlier, surgically treated, presented with a sudden onset, one day before, of posterior headache with neck pain, without any effort or neck trauma, followed by dizziness, vertigo and nausea. Neurological examination on admission was poor, with only mild dysmetria.

A CT scan, performed in the Emergency Department, showed an infarction of the right cerebellar hemisphere. Afterwards emergency MR angiography revealed right VA dissection and occlusion of the right posterior inferior cerebellar artery (PICA). Administration of antithrombotic agents (heparin first, followed by dicumarolics) was immediately started, with a rapid recovery.

A few days after discharge, the patient presented with an acute episode of objective vertigo with nystagmus, which disappeared a few hours later, after flunarizine treatment. A CT scan was negative for new lesions.

\section{Discussion}

Headache or facial or neck pain attributed to CAD is a secondary headache described in chapter 6 of the 2004 IHS Classification of vascular disorders [4].

Headache or neck pain are often the only manifestation of CAD; it is by far the most frequent symptom and often the inaugural one. The characteristics of this headache can be very misleading, mimicking other primary headaches, such as migraine, primary thunderclap headache, or other secondary ones, such as post-traumatic headache (if a neck trauma history or whiplash is present) or subarachnoid haemorrhage. Therefore, an accurate neurological examination is mandatory, particularly in young adults, in order to search for symptoms or local signs of cerebral stroke; MRI/MRA and CT angiography are useful non-invasive diagnostic tests, necessary for an early diagnosis. The accepted management of spontaneous CAD with cerebral infarction and no subarachnoid haemorrhage consists of anticoagulant therapy [3], although controlled studies to show its effectiveness are lacking. In some cases of recurrent thromboembolism despite maximal anticoagulation therapy, surgical treatment can be suggested [5]. However there still remains an open question as to how therapy should proceed in the long term; further studies are necessary, even though the literature data show that recurrences are rare in CAD.

\section{References}

1. Thanvi B, Munshi SK, Dawson SL, Robinson TG (2005) Carotid and vertebral artery dissection syndromes. Postgrad Med J 81:383-388

2. Lelong DC, Logak M (2004) Pathogenesis of spontaneous cer- 
vico-cerebral artery dissection. A hypothesis and a review of the literature. Med Hypotheses 62:453-457

3. Dziewas R, Konrad C, Drager B, Evers S, Besselmann M, Ludemann P, Kuhlenbaumer G, Stogbauer F, Ringelstein EB (2003) Cervical artery dissection-clinical features, risk factors, therapy and outcome in 126 patients. J Neurol 250:1179-1184
4. Headache Classification Committee of the International Headache Society (2004) The International Classification of Headache Disorders, 2nd edn. Cephalalgia 24[Suppl 1]:1-160

5. Chiche L, Praquin B, Koskas F, Kieffer E (2005) Spontaneous dissection of extracranial vertebral artery: indications and long-term outcome of surgical treatment. Ann Vasc Surg 19:5-10 